\title{
Discrete matrix Schrödinger equation equivalents of discrete two-component matrix wave systems
}

\author{
Andrew E Yagle \\ Department of Electrical Engineering and Computer Science, University of Michigan, \\ Ann Arbor, MI 48109-2122, USA
}

Received 27 July 1988, in final form 1 February 1989

\begin{abstract}
Multichannel discrete lossless two-component wave systems describe physical scattering for multiple coupled transmission lines, for elastic media, and for electromagnetic wave propagation in layered media. Such a wave system is transformed into an equivalent discrete matrix Schrödinger equation that includes the effects of transmission losses and transmission scattering. These effects are not included in the continuous Schrödinger equation, so that discretisation of the latter omits significant effects. Applications include fast algorithms for synthesis and inversion of the reflection responses of multichannel scattering media. These algorithms require roughly half as many matrix multiplications as previous algorithms that employ the multichannel two-component wave system.
\end{abstract}

\section{Introduction}

Multichannel discrete lossless two-component wave systems describe scattering in a wide variety of physical settings. These include multiple coupled transmission lines, electromagnetic wave propagation in layered media [1], and wave propagation in elastic media [1-3]. All of these problems are commonly formulated in terms of the multichannel wave system shown in figure 1 . In this system $p$ waves travelling in one direction are reflected into $p$ waves travelling in the opposite direction. In addition, the waves travelling in a given direction are scattered into each other, and conservation of energy for a lossless medium requires that the unscattered waves be attenuated to reflect the energy lost by scattering.

For such multichannel scattering media, the two major problems of interest are the synthesis of the reflection response to an impulse in any of the downgoing waves, and the inverse problem of reconstructing the medium from these reflection responses. The synthesis problem is generally solved using the reflectivity method $[4,5]$, which propagates the multichannel two-component wave system backwards. The inverse problem for multichannel media is solved most efficiently using a layer-stripping approach, which recursively reconstructs the reflection coefficients and propagates the multichannel two-component wave system forward into the medium. This approach was used for elastic media in $[6,7]$.

An alternative for the inverse problem is to employ a continuous model for the multichannel scattering medium. This has the effect of neglecting the transmission losses and transmission scattering, since these are second-order effects. This approach 


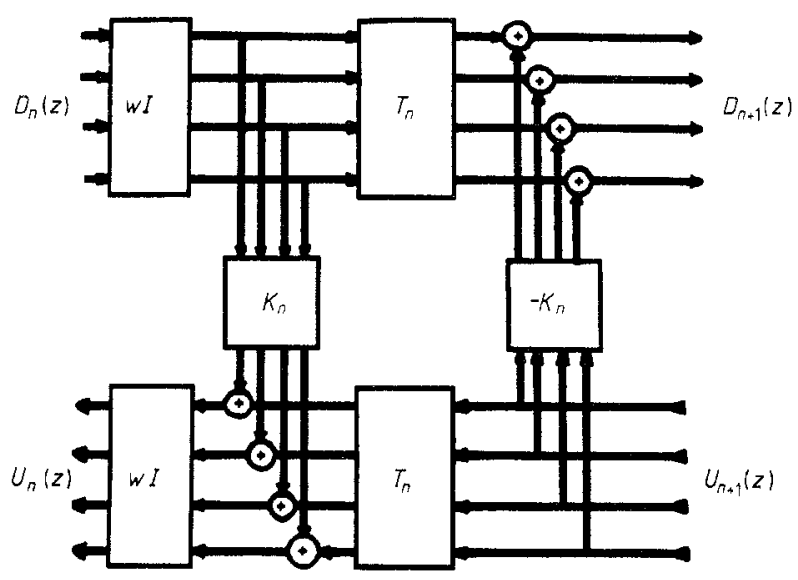

Figure 1. Wave propagation in a multichannel scattering medium. The wave in the $i$ th channel is scattered into the opposite-travelling wave in the $j$ th channel with reflection coefficient $k_{i j}=k_{j i}$. The matrix of refiection coefficients at the $n$th interface is $K_{n}=\left[k_{i j}\right]$.

was used in [8-11]. However, the neglect of the transmission effects must lead to errors, since the discretised form of the continuous equations is not lossless. In particular, the discretised matrix Schrödinger equation in [10] and matrix Riccati equation in [11] neglect these effects.

This paper transforms the multichannel two-component wave system of figure 1 into a discrete matrix Schrödinger equation that includes the effects of transmission losses and transmission scattering in its potential. This result, which seems to be new, is then used to derive new fast algorithms for the reflection response synthesis and inversion problems for multichannel scattering media, including elastic and electromagnetic scattering problems. These algorithms include all transmission effects, but require only roughly half as many matrix multiplications as previous algorithms that employ two-component wave systems.

This paper is organised as follows. Section 2 introduces the multichannel twocomponent wave system, and two different discrete matrix Schrödinger equations are derived from it (the actual derivations are relegated to an appendix). Section 2 also discusses how various solutions of the two-component system map into solutions of the matrix Schrödinger equation. Section 3 derives the fast algorithms for the reflection response synthesis and inversion problems. Section 4 concludes with a summary of results and directions for further research.

\section{Discrete Schrödinger equation equivalents of multichannel wave systems}

In this section the multichannel two-component wave system is discussed and motivated briefly. Two different discrete matrix Schrödinger equations are derived from it (the actual derivations are relegated to an appendix); these are generalisations of different discrete scalar Schrödinger equations. The wave system quantitites propagated by the multichannel Levinson and Schur algorithms are mapped into the matrix regular and scattering solutions of the discrete Schrödinger equation. The multichannel Schur formula, which is the proper discretisation of the matrix Riccati equation used in $[11,12]$, is also noted. 


\subsection{Multichannel two-component wave systems}

The multichannel two-component wave system illustrated in figure 1 is described mathematically as follows. For an isotropic medium, the reflection coefficient $k_{i j}$ for the $i$ th downgoing wave reflected into the $j$ th upgoing wave must be equal to $k_{j i}$. The property $k_{i j}=k_{j i}$ implies that the reflection coefficient matrix at the $n$th interface $K_{n}=\left[k_{i j}\right]$ is symmetric. The reflection coefficients for upgoing waves reflected into downgoing waves are $-k_{i j}$, since the wave effects on the medium are seen by these waves to act in the opposite direction or to have opposite phase. Finally, by isotropy the transmission matrices for downgoing and upgoing waves must be equal. Let the transmission matrix at the $n$th interface be $T_{n}$.

The above discussion shows that the waves $D_{n}(-, t)$ and $U_{n}(-, t)$ just above the $n$th interface are related to the waves $D_{n}(+, t)$ and $U_{n}(+, t)$ just below the $n$th interface by the scattering matrix

$$
\left[\begin{array}{l}
D_{n}(+, t) \\
U_{n}(-, t)
\end{array}\right]=\left[\begin{array}{cc}
T_{n} & -K_{n} \\
K_{n} & T_{n}
\end{array}\right]\left[\begin{array}{l}
D_{n}(-, t) \\
U_{n}(+, t)
\end{array}\right]
$$

Conservation of energy requires that this matrix be unitary, which implies that

$$
T_{n}=\left(I-K_{n}^{2}\right)^{1 / 2}
$$

which includes not only transmission losses but transmission scattering between different waves travelling in the same direction.

Equations (2.1) and (2.2) can be rearranged into a form exhibiting the layer matrix

$$
\left[\begin{array}{c}
D_{n}(+, t) \\
U_{n}(+, t)
\end{array}\right]=\left(I-K_{n}^{2}\right)^{-1 / 2}\left[\begin{array}{cc}
I & -K_{n} \\
-K_{n} & I
\end{array}\right]\left[\begin{array}{c}
D_{n}(-, t) \\
U_{n}(-, t)
\end{array}\right]
$$

where the factor $\left(I-K_{n}^{2}\right)^{-1 / 2}$ multiplies each of two components. The layer matrix (2.3) can be found in each of [1-3], where it describes elastic or electromagnetic wave propagation across an interface. Considerable details on the derivation of (2.3) for elastic media are available in [2]. For isotropic elastic media $K_{n}$ is $2 \times 2$; certain types of anisotropic elastic media can be treated by augmenting the wave system, so that $K_{n}$ is a larger matrix [3, 9].

We assume constant wave speed, which without loss of generality may be taken to be unity. Electromagnetic plane wave propagation can be formulated in this way [1]. The varying wave speeds for elastic waves are handled in $\$ 3$ below. If $z$ is the two-way unit delay operator, then the $z$ transforms of the waves $D_{n}(z)$ and $U_{n}(z)$ just above the $n$th interface satisfy

$$
\left[\begin{array}{l}
D_{n+1}(z) \\
U_{n+1}(z)
\end{array}\right]=\frac{1}{w}\left(I-K_{n}^{2}\right)^{-1 / 2}\left[\begin{array}{cc}
z I & -K_{n} \\
-z K_{n} & I
\end{array}\right]\left[\begin{array}{l}
D_{n}(z) \\
U_{n}(z)
\end{array}\right]
$$

where $w=z^{1 / 2}$ is the one-way unit delay operator. Equation (2.4) is the defining equation for the multichannel two-component wave system described in figure 1.

In the following we will often drop the factor of $w$ in $(2.4)$, so that $D_{n}(z)$ and $U_{n}(z)$ are polynomials in $z$. This factor simply scales the waves by $z^{n / 2}$, which advances them so that polynomial coefficients known by causality to be zero need not be stored. 


\subsection{Matrix Schrödinger equations}

In the appendix it is shown that the system (2.4) can be transformed into the discrete matrix Schrödinger equation

$$
S_{n+1}(z)=(w+1 / w) S_{n}(z)-V_{n-1} S_{n-1}(z)
$$

where $S_{n}(z)$ and the discrete scattering potential $V_{n}$ are defined by

$$
\begin{aligned}
& S_{n}(z)=T_{n}\left(I-K_{n-1}\right)^{-1}\left(D_{n}(z)+U_{n}(z)\right) \\
& V_{n}=T_{n}\left(I+K_{n}\right)\left(I-K_{n-1}\right) T_{n}^{-1} \\
& T_{n}=\prod_{i=1}^{n-1}\left(I-K_{i}^{2}\right)^{1 / 2} .
\end{aligned}
$$

In $(2.6 c)$ the product is taken in the order $i=1$ leftmost, $i=n-1$ rightmost. Note that $T_{n}$ in $(2.6 c)$ is now the cumulative transmission effect of the transmission coefficients defined in (2.2). Note also that $I-V_{n-1}$ is the actual quantity that determines scattering in (2.5); for convenience we refer to $V_{n}$ as the potential.

Equation (2.5) has the form of the obvious discretisation of a continuous Schrödinger equation. However, $S_{n}(z)$ in $(2.6 a)$ is normalised by the cumulative transmission effect $T_{n}$, which is not included in a discretisation of the continuous version of the system (2.4). Furthermore, the potential $V_{n}$ is pre-normalised by $T_{n}$ and post-normalised by $T_{n}^{-1}$, two factors which cancel in the scalar version of these equations and thus cannot be foreseen from their scalar development in [13].

A minor change in the last step of the development in the appendix results in the different discrete matrix Schrödinger equation

$$
S_{n+1}(z)=(w+1 / w) W_{n} S_{n}(z)-S_{n-1}(z)
$$

where $S_{n}(z)$ and the matrix of slownesses $W_{n}$ are here defined by

$$
\begin{aligned}
& S_{n}(z)=L_{n} Q_{n}\left(D_{n}(z)+U_{n}(z)\right) \\
& W_{n}=L_{n-1} Q_{n-1}\left(I+K_{n-1}\right)^{-1} Q_{n-1}^{-1} L_{n}^{-1} \\
& Q_{n}=\prod_{i=1}^{n-1}\left(I-K_{i}\right)^{-1}\left(I-K_{i}^{2}\right)^{1 / 2} \\
& L_{n+1}=L_{n-1}\left[Q_{n-1}\left(I+K_{n-1}\right)^{-1}\left(I-K_{n-1}\right) Q_{n}^{-1}\right] .
\end{aligned}
$$

The scalar forms of these equations were derived in [13]; the multichannel generalisations obviously have many features that cannot be foreseen from the scalar development.

It is difficult to attach physical interpretations to (2.8); however, $(2.7)$ is a matrix version of the classical discrete Schrödinger equation treated in [14]. Note that this is actually a discrete matrix wave equation, in which $W_{n}$ is a matrix of slownesses; the scattering effect is produced by variations of $W_{n}$.

Equation (2.7) is also interesting in that it has the same form as the three-term recurrence for the matrix singular predictor polynomials of [15], if $S_{n}(z)$ is scaled by $z^{n / 2}$. This is a matrix version of the split or immittance-domain algorithms, which have appeared in the signal processing literature recently $[16,17]$. In fact, viewed in this light (2.7) is a matrix generalisation of the balanced recurrences, while (2.5) is a matrix generalisation of the monic recurrences [18]. However, the inclusion of 
multichannel transmission effects results in the complicated expressions (2.6) and (2.8) not present in the scalar case.

\subsection{Mapping of solutions}

2.3.1. Scattering solution. Suppose that the system (2.4) is initialised with

$$
D_{1}(z)=I \quad U_{1}(z)=R(z)
$$

where $R(z)$ is the $z$ transform of the matrix reflection response of the system (2.4) to impulses. Specifically, the $i j$ th element of $R(z)$ is the response in the $i$ th upgoing channel of (2.4) to an impulse in the $j$ th downgoing channel, with all other downgoing channels initialised to zero. Then the $i j$ th elements of $D_{n}(z)$ and $U_{n}(z)$ are the waves above the $n$th interface in the $i$ th channels resulting from the impulse in the $j$ th channel (note that for all quantities different columns correspond to different experiments, while rows are associated with specific channels).

By causality $U_{n+1}(z) /\left.w^{n+1}\right|_{z=0}=0$. Then from (2.4) we have that

$$
K_{n}=\left.U_{n}(z) D_{n}(z)^{-1 / z}\right|_{z=0} .
$$

Hence the inverse problem for (2.4) may be solved as follows. Given the matrix reflection response $R(z)$, initialise (2.4) with (2.9) and propagate (2.4) in increasing $n$, recovering $K_{n}$ from (2.10) along the way. This is the multichannel Schur or layerstripping algorithm.

Now associate the discrete Schrödinger equation (2.5) with the two-component system (2.4), where the quantities propagated by $(2.4)$ are the waves associated with the Schur algorithm. Then the $S_{n}(z)$ propagated in (2.5) is the scattering solution [19] of the discrete Schrödinger equation. This is the solution determined by the initial condition $S_{1}(z)=I+R(z)$, with a radiation condition as $n \rightarrow \infty$. using

The scattering potential $V_{n-1}$ can be obtained from the scattering solution $S_{n}(z)$ by

$$
V_{n-1}=S_{n}(z) S_{n-1}^{-1}(z) /\left.w\right|_{z=0}
$$

since $S_{n+1}(z) /\left.w^{n}\right|_{z=0}=w S_{n}(z) /\left.w^{n}\right|_{z=0}=0$ by causality. The scalar version of (2.11) appears in the split Schur algorithm of [17].

\subsubsection{Regular solution. Now let the system (2.4) be initialised with}

$$
D_{1}(z)=I \quad U_{1}(z)=0 .
$$

Now the $2 p \times p$ matrix $\left[D_{n}^{\mathrm{T}}(z) U_{n}^{\mathrm{T}}(z)\right]^{\mathrm{T}}$ propagated by $(2.4)$ is the first column of the state transition matrix associated with the discrete time system (2.4) (the second column is $\left.z^{n}\left[U_{n}^{\mathrm{T}}(1 / z) D_{n}^{\mathrm{T}}(1 / z)\right]^{\mathrm{T}}\right)$.

Using this property and (2.10) shows that the reflection coefficients $K_{n}$ may be computed from the reflection response $R(z)$ and the $D_{n}(z)$ resulting from initialisation with (2.12). We have

$$
K_{n}=D_{n}(z) R(z) T_{n}^{-1} /\left.z^{n}\right|_{z=0}
$$

where $T_{n}$ is defined in (2.6c). Hence another solution to the inverse problem for (2.4) is as follows. Initialise (2.4) with (2.12) and propagate (2.4) and (2.6c) in increasing $n$, recovering $K_{n}$ from (2.13) along the way. This is the multichannel Levinson or layer-adjoining algorithm. 
Now associate the discrete Schrödinger equation (2.5) with the two-component system (2.4), where the quantities propagated by (2.4) are the waves associated with the Levinson algorithm. Then the $S_{n}(z)$ propagated in $(2.5)$ is the regular solution [19] of the discrete Schrödinger equation. This is the solution determined by the initial condition $S_{1}(z)=I$; this implies that the regular solution is the discrete Green function for the Schrödinger equation (2.5).

\subsection{Comments}

The association of the Levinson and Schur algorithms of signal processing with the layer-stripping and layer-adjoining inverse scattering algorithms is discussed in more detail in the scalar case in [20]. Much of this association for the multichannel case appears in disguised form in [2], although there is no mention of fast algorithms there.

The matrix three-term recursions derived in [15] are valid for non-symmetric $K_{n}$. However, there is no computational savings in the three-term recurrences of [15] unless the $W_{n}$ and $K_{n}$ are symmetric. Fortunately, this is the case of physical interest, and this makes possible the fast algorithms of $\$ 3$ below. Also, the three-term recurrences of [15] can be interpreted as discrete Schrödinger equations only if the $W_{n}$ are symmetric, and the recurrences of [15] did not include the transmission scattering and losses included in (2.5) and (2.7).

Equations (2.6) and (2.8) are much more complicated than the corresponding scalar equations in [13]. This is because transmission effects can be ignored entirely in the scalar case-the effects of the losses going down and up cancel each other out. Indeed, note that in the scalar case $(2.6 b)$ becomes

$$
V_{n}=\left(I+K_{n}\right)\left(I-K_{n-1}\right)
$$

which appears both in [13] for the scalar discrete Schrödinger equation, and in [17] for the scalar split algorithms. Also note that the mapping from (2.4) to (2.5) is unique, but the reverse mapping is not, since $K_{0}$ can be chosen to have different values. This is discussed in detail for the scalar case in [13].

\subsection{Schur formula and Riccati equations}

Another procedure is as follows. Defining the reflection response at depth

$$
R_{n}(z)=U_{n}(z) D_{n}^{-1}(z) / z
$$

it is straightforward to show from $(2.4)$ that $R_{n}(z)$ satisfies the matrix Schur formula

$$
R_{n+1}(z)=\frac{1}{z}\left(I-K_{n}^{2}\right)^{-1 / 2}\left(R_{n}(z)-K_{n}\right)\left(I-K_{n} R_{n}(z)\right)^{-1}\left(I-K_{n}^{2}\right)^{1 / 2} .
$$

Equation (2.16) constitutes an alternative to the multichannel Schur algorithm. Propagate (2.16) in increasing $n$, initialised with $R_{1}(z)=R(z)$, and recovering $K_{n}$ using $K_{n}=R_{n}(0)$ (see $(2.10)$ and $(2.15)$ ) along the way.

Equation (2.16) is a discrete version of the matrix Riccati equation used in [11] for reconstructing elastic media and in [12] for coupled transmission lines. To see this, let the layers each have thickness $\Delta$, so that the two-way unit delay operator $z=\exp (-2 \mathrm{i} \omega \Delta)$. Since $K_{n}$ is proportional to $\Delta$ by $K_{n}=K(n \Delta) \Delta$, the transmission effects $\left(I-K_{n}^{2}\right)^{1 / 2}$ are $\mathrm{O}\left(\Delta^{2}\right)$ and negligible as $\Delta \rightarrow 0$. Post-multiplying (2.16) by the 
inverted term and letting $\Delta \rightarrow 0$ results in the matrix Riccati equation in depth $x$ and frequency $\omega$

$$
\frac{\mathrm{d} R}{\mathrm{~d} x}(x, \omega)=2 \mathrm{i} \omega R-K(x)+R K R
$$

which is comparable to the matrix Riccati equations in $[11,12]$ (there are some differences due to varying layer wave speeds).

Unlike the Riccati equation (2.17), (2.16) includes all transmission loss and transmission scattering effects (in the first and last terms, which cancel in the scalar case). Note that multiple reflections are accounted for in a very elegant manner in (2.16) by incorporating a feedback correction term with the loop gain $K_{n} R_{n}(z)$.

It is also worth noting that the same discretisation applied to $(2.6 b)$ results in the matrix version of the Miura transform [13]

$$
V(x)=K(x)^{2}-d K(x) / d x
$$

where $V(n \Delta) \Delta^{2}=I-V_{n}$ is the continuous scattering potential.

\section{New fast algorithms for synthesis and inversion}

In this section new fast algorithms for the multichannel synthesis and inversion problems are derived, based on the discrete Schrödinger equation (2.5). These are suitable for coupled transmission lines and electromagnetic wave propagation through layered media. For elastic wave propagation, the different wave speeds for $\mathrm{P}$ and $\mathrm{SV}$ waves requires some special treatment (see $\$ 3.3$ ).

\subsection{Fast algorithm for inversion}

This algorithm consists of propagating the scattering solution $S_{n}(z)$ to the discrete Schrödinger equation (2.5), recovering the potential $V_{n}$ using (2.11). The reflection coefficients $K_{n}$ are then recovered from the potential $V_{n}$ using the relations $(2.6 b)$ and (2.6c). The only difference is that $S_{n}(z)$ is scaled by $w^{n}$ (corresponding to omitting the $1 / w$ in $(2.4)$ ) to eliminate half-powers of $z$.

(i) Initialisation

$$
S_{1}(z)=I+R(z) \quad S_{2}(z)=z I+R(z) \quad K_{1}=0 .
$$

(ii) Propagation in increasing $n$

$$
\begin{aligned}
& S_{n+1}(z)=(z+1) S_{n}(z)-z V_{n-1} S_{n-1}(z) \\
& V_{n-1}=S_{n}(z) S_{n-1}^{-1}(z) /\left.z\right|_{z=0} .
\end{aligned}
$$

(iii) Recovery of $K_{n}$ from $V_{n}$

$$
\begin{aligned}
& T_{n}=T_{n-1}\left(I-K_{n-1}^{2}\right)^{1 / 2} \\
& K_{n}=T_{n}^{-1} V_{n} T_{n}\left(I-K_{n-1}\right)^{-1}-I .
\end{aligned}
$$

Equations (3.2) and (3.3) are obtained from (2.5) and (2.11) by scaling $S_{n}(z)$ with $w^{n}$. Equation (3.4) recuisively implements $(2.6 c)$, while (3.5) inverts $(2.6 b)$. This inversion is unique provided that we know a priori that there is no immediate reflection, i.e. that $R(0)=0$. This implies that $K_{1}=0$. Otherwise, we must know $K_{1}$ to 
initialise (3.5). Since the reflection response is known to arise from a physical medium, the reconstructed sequence of $K_{n}$ will not become unstable. Noise in the reflection response may cause trouble here, but this is also possible with the mutichannel Schur algorithm.

The propagation of the scattering solution to (2.5) is equivalent to propagating the field quantities in the physical scattering medium, rather than the waves of (2.4). For coupled transmission lines, the field quantities are the voltages or currents in each line, energy-normalised by their characteristic impedances. For elastic media, the field quantities are the normal and shear stresses, or the components of medium displacement, again energy-normalised by their impedances. The energy normalisation is a good physical indication of the numerical stability of (3.2).

The reason for preferring this algorithm over the multichannel Schur algorithm (2.4) and (2.10) is that the Schur algorithm requires at each recursion multiplication of $K_{n}$ by each coefficient of $D_{n}(z)$ and $U_{n}(z)$. If $N$ coefficients are stored, this amounts to $2 N$ matrix multiplications per recursion for this multiplication. And this does not include (2.10) or the computation of and multiplication by $\left(I-K_{n}^{2}\right)^{-1 / 2}$.

On the other hand, (3.2) requires only $N$ matrix multiplications per recursion, since the single matrix polynomial $S_{n}(z)$ replaces $D_{n}(z)$ and $U_{n}(z)$. Equations (3.3) and (3.4) have their direct computational counterparts in the multichannel Schur algorithm. The only additional computation is (3.5), and for large $N$ this multiplication is negligible. Hence there is a saving of roughly $50 \%$ (slightly less due to (3.5)) in matrix multiplications.

\subsection{Fast algorithm for synthesis}

The reflectivity method $[4,5]$ solves the synthesis problem for a medium of $N$ layers as follows. Initialise (2.4) with $D_{N}(z)=I ; U_{N}(z)=K_{N}$, and propagate (2.4) backwards in decreasing $n$, using the known values of $K_{n}$ along the way. By linearity, the reflection response is then given by $R(z)=U_{1}(z) D_{1}^{-1}(z)$.

The fast algorithm for the synthesis problem consists of propagating (2.5) backwards, where $S_{n}(z)$ now is associated with the $D_{n}(z)$ and $U_{n}(z)$ propagated by the reflectivity method. Again $S_{n}(z)$ is scaled by $w^{n}$.

(i) Propagation in increasing $n$

$$
\begin{aligned}
& T_{n}=T_{n-1}\left(I-K_{n-1}^{2}\right)^{1 / 2} \\
& V_{n}=T_{n}\left(I+K_{n}\right)\left(I-K_{n-1}\right) T_{n}^{-1} .
\end{aligned}
$$

(ii) Initialisation

$$
\begin{aligned}
& S_{N}(z)=T_{N}\left(I-K_{N-1}\right)^{-1}\left(I+K_{N}\right) \\
& S_{N+1}(z)=\left(I-K_{N}^{2}\right)^{-1 / 2}\left(I-K_{N}\right)\left(S_{N}(z)+(z-1) I\right) .
\end{aligned}
$$

(iii) Propagation in decreasing $n$

$$
S_{n-1}(z)=V_{n-1}^{-1}\left((z+1) S_{n}(z)-S_{n+1}(z)\right) / z .
$$

(iv) Recovery of $R(z)$

$$
R(z)=-\left(S_{2}(z)-z S_{1}(z)\right)\left(S_{2}(z)-S_{1}(z)\right)^{-1} .
$$


Equations (3.6) and (3.7) are propagated in increasing $n$ to precompute the $V_{n}$ from the known $K_{n}$. This is necessary unless $T_{N}$ is already known. The initialisation $(3.8 a)$ follows directly from (2.6a) and the initialisation of the reflectivity method; $(3.8 b)$ follows directly from (A2a) in the appendix, as does $(3.10) ;(3.9)$ is just (2.5) propagated backwards. In (3.10) we again assume $K_{1}=0$; if not, some extra factors are present (see (A2)).

As in the fast algorithm for the multichannel inverse problem, the computational saving is a reduction of roughly $50 \%$ in the number of matrix multiplications, since the single matrix polynomial $S_{n}(z)$ replaces $D_{n}(z)$ and $U_{n}(z)$. The $V_{n}$ must be precomputed in increasing $n$ first; however, the $K_{n}$ must be computed ahead of time for the reflectivity method, and the $V_{n}$ may overwrite the $K_{n}$.

It is worth noting that the computational savings in these algorithms also apply in the scalar case, so that they are applicable to single-channel (e.g. acoustic) scattering media. The $50 \%$ saving in multiplications still holds, and the transmission terms all cancel out, which simplifies matters greatly.

\subsection{Varying wave speeds: the elastic case}

For the special case of an elastic medium the wave speeds of the two channels (compressional $P$ waves and shear SV waves) differ. This is unfortunate, since there seems to be no way to modify the derivation in the appendix to incorporate different wave speeds in different channels. However, for elastic media consisting of many thin layers, it is possible to incorporate two different wave speeds by modifying an idea noted in [2].

Let the medium be divided up into equal P-wave travel time layers, thin enough so that only an occasional layer corresponds to an actual medium interface. If the layers are thin enough, all $\mathrm{P}$ and $\mathrm{Sv}$ wave reflections will occur after passage through an integral number of these layers. In other words, the time delay between interfaces for both wave types can be regarded as passage through an integral number of layers, with different integers for each wave type. The same approach was taken in [2]. Note that in any algorithm time must ultimately be discretised, which results in precisely this situation anyway; we are merely being more explicit here.

This modification allows the above algorithm to be used for the elastic medium reflection response synthesis problem. The $K_{n}$ are now associated with each layer, not with each interface; most of the $K_{n}$ will be zero, requiring no computation. Some $K_{n}$ will have SV and mode conversion elements, but no P elements; some will have $P$ and mode conversions elements, but no sv elements. And some will have all types of elements.

If the actual medium has many thin layers, then the above algorithm will be useful, especially since the cumulative transmission effects become more important as the number of layers increases. For media with few interfaces, the number of non-zero $K_{n}$ will be much greater than the actual number of interfaces, and the above algorithm may require more computation than the standard reflectivity method.

In applying the new algorithms to variable-velocity media, it is necessary that there be no turning points or degeneracies in the medium, since otherwise the assumption of propagating waves described by (2.4) no longer holds. For media with different wave speeds in different channels, the discretisation used above will eventually result in significant errors, since reflections will be occurring at the wrong times. However, finite precision in numerical computation will eventually cause this effect anyway. 


\section{Conclusion}

Two discrete matrix Schrödinger equations were derived from the multichannel lossless two-component wave system. These two matrix Schrödinger equations were generalisations of similar scalar equations that had appeared in $[13,14]$, and of the split signal processing algorithms of [15-18]. However, the matrix Schrödinger equations derived here incorporate all transmission losses and transmission scattering between channels. This is in contrast to the continuous matrix Schrödinger equation, which is easier to derive but which includes none of these effects. These effects also cancel out in the scalar case considered in [13], so that the generalisation to the multichannel case is not trivial. Mappings between the Levinson and Schur algorithms, and the regular and scattering solutions to the matrix Schrödinger equation, were also noted.

Fast algorithms for synthesising the reflection response of a layered medium, and for reconstructing the medium from its reflection response, were derived using the new Schrödinger equation. These algorithms require approximately half the number of matrix multiplications required by the wave-system based algorithms. Although derived for the matrix case, they are equally useful for the scalar case, e.g. for acoustic media. A possible application to elastic media was also noted.

Unsolved issues include studying the inverse of the transformation (2.6), in particular to determining necessary and sufficient conditions for a potential sequence $V_{n}$ to correspond to a stable set of reflection coefficients $K_{n}$. The scalar version of this problem was treated in [13]. Modifications of the discrete Schrödinger equation that allow varying wave speeds between channels would be directly applicable to elastic media. And the numerical properties of the algorithms proposed here need to be studied.

\section{Acknowledgment}

This research was supported by the National Science Foundation under grant MIP-8858082.

\section{Appendix}

This appendix contains the details of the derivations of the discrete matrix Schrödinger equations (2.5) and (2.7) from the matrix two-component wave system (2.4).

Defining $S_{n}(z)=D_{n}(z)+U_{n}(z)$, we have from (2.4) that

$$
\begin{aligned}
S_{n+1}(z)=\left(I-K_{n}^{2}\right)^{-1 / 2}\left(I-K_{n}\right)\left[S_{n}(z)+(z-1) D_{n}(z)\right] \\
\quad=\left(I-K_{n}^{2}\right)^{-1 / 2}\left(I-K_{n}\right)\left[z S_{n}(z)+(1-z) U_{n}(z)\right] .
\end{aligned}
$$

Solving (A1) for $D_{n}(z)$ and $U_{n}(z)$ gives

$$
\begin{aligned}
& D_{n}(z)=\left(I-K_{n}\right)^{-1}\left(I-K_{n}^{2}\right)^{1 / 2}\left(S_{n+1}(z)-S_{n}(z)\right) /(z-1) \\
& U_{n}(z)=\left(I-K_{n}\right)^{-1}\left(I-K_{n}^{2}\right)^{1 / 2}\left(S_{n+1}(z)-z S_{n}(z)\right) /(1-z)
\end{aligned}
$$


and inserting (A2) into the bottom row of (2.4) and multiplying by $(1-z)$ gives

$$
\begin{aligned}
\left(I-K_{n}\right)^{-1}(I- & \left.K_{n}^{2}\right)^{1 / 2}\left(S_{n+1}(z)-z S_{n}(z)\right) \\
& =\left(I-K_{n-1}^{2}\right)^{-1 / 2}\left[\left(I-K_{n-1}\right)^{-1}\left(I-K_{n-1}^{2}\right)^{1 / 2}\left(S_{n}(z)-z S_{n-1}(z)\right)\right. \\
& \left.+z K_{n-1}\left(I-K_{n-1}\right)^{-1}\left(I-K_{n-1}^{2}\right)^{1 / 2}\left(S_{n}(z)-S_{n-1}(z)\right)\right] .
\end{aligned}
$$

Now define $S_{n}^{\prime}(z)$ by

$$
\begin{aligned}
& S_{n}(z)=P_{n} S_{n}^{\prime}(z) \\
& P_{n}=\prod_{i=1}^{n-1}\left(I-K_{i}^{2}\right)^{-1 / 2}\left(I-K_{i}\right)
\end{aligned}
$$

where the $i=n-1$ term is leftmost and the $i=1$ term is rightmost. Substitute (A4) into (A3), pre-multiply by $\left(I-K_{n-1}^{2}\right)^{1 / 2}$, and cancel the $z K_{n-1} P_{n-1} S_{n}^{\prime}(z)$ term. This gives

$\left(I-K_{n-1}\right) P_{n-1} S_{n+1}^{\prime}(z)=(z+1) P_{n-1} S_{n}^{\prime}(z)-z\left(I+K_{n-1}\right) P_{n-1} S_{n-1}^{\prime}(z)$

and pre-multiplying by $P_{n-1}^{-1}\left(I-K_{n-1}\right)^{-1}$ gives

$$
\begin{aligned}
S_{n+1}^{\prime}=(z+1) & P_{n-1}^{-1}\left(I-K_{n-1}\right)^{-1} P_{n-1} S_{n}^{\prime}(z) \\
& -z P_{n-1}^{-1}\left(I-K_{n-1}\right)^{-1}\left(I+K_{n-1}\right) P_{n-1} S_{n-1}^{\prime}(z)
\end{aligned}
$$

To derive (2.5) define $S_{n}^{\prime \prime}(z)$ by

$$
S_{n}^{\prime}(z)=L_{n} S_{n}^{\prime \prime}(z)
$$

where $L_{n}$ is defined to simplify the centre term of (A7), i.e.

$$
L_{n+1}=P_{n-1}^{-1}\left(I-K_{n-1}\right)^{-1} P_{n-1} L_{n} .
$$

It is easy to see that $L_{n}$ is given by

$$
\begin{aligned}
& L_{n}=P_{n-1}^{-1} T_{n-1} \\
& T_{n}=\prod_{i=1}^{n-1}\left(I-K_{i}^{2}\right)^{-1 / 2}
\end{aligned}
$$

where again the $i=n-1$ term is leftmost and the $i=1$ term is rightmost.

Substituting (A7) and (A8) into (A6) results in

$$
S_{n+1}^{\prime \prime}(z)=(z+1) S_{n}^{\prime \prime}(z)-z V_{n-1} S_{n-1}^{\prime \prime}(z)
$$

where the potential $V_{n}$ is defined from (A6) as

$$
\begin{array}{r}
V_{n-1}=L_{n+1}^{-1} P_{n-1}^{-1}\left(I-K_{n-1}\right)^{-1}\left(I+K_{n-1}\right) P_{n-1} L_{n-1} \\
=T_{n-1}^{-1}\left(I+K_{n-1}\right)\left(I-K_{n-2}\right) T_{n-1} .
\end{array}
$$

The identity $\left(I-K_{n-2}^{2}\right)^{-1 / 2} K_{n-2}=K_{n-2}\left(I-K_{n-2}^{2}\right)^{-1 / 2}$, valid for symmetric $K_{n-2}$, is used in simplifying (A11). Also using this identity, (A4) and (A7) combine into

$$
S_{n}(z)=\left(I-K_{n-1}\right) T_{n} S_{n}^{\prime \prime}(z) \text {. }
$$

Equations (A10)-(A12) lead directly to (2.5) and (2.6), except that the definitions used in $\S 2$ are the matrix inverses of those used here.

Equation (2.7) is derived similarly, except that (A8) is replaced with

$$
L_{n+1}=P_{n-1}^{-1}\left(I-K_{n-1}\right)^{-1} P_{n-1} L_{n-1}
$$


in order to simplify the last term of (A7) instead of the centre term. This clearly results in an equation having the form of (2.7), although $W_{n}$ has a more complex definition (2.8) than $V_{n}$.

\section{References}

[1] Ursin B 1983 Review of elastic and electromagnetic wave propagation in horizontally layered media Geophysics 48 1063-81

[2] Frasier C W 1970 Discrete-time solution of plane P-SV waves in a plane layered medium Geophysics 35 197-219

[3] Kennett B L N 1983 Seismic Wave Propagation in Stratified Media (Cambridge: Cambridge University Press)

[4] Aki K and Richards P G 1980 Quantitative Seismology, Theory and Methods (San Francisco: Freeman) p 393

[5] Kind R 1976 Computation of reflection coefficients for layered media J. Geophys. 42 191-200

[6] Shiva M and Mendel J M 1983 Non-normal incidence inversion: existence of solution Geophys. Prosp. 31 888-914

[7] Clarke T J 1984 Full reconstruction of a layered elastic medium from P-sv slant stack data Geophys. $J$. R. Astron. Soc. 78 775-93

[8] Yagle A E and Levy B C 1985 A layer stripping solution of the inverse problem for a one-dimensional elastic medium Geophysics $\mathbf{5 0}$ 425-33

[9] Meadows M and Coen S 1986 Exact inversion of plane-layered isotropic and anisotropic elastic media by the state-space approach Geophysics $\mathbf{5 1} 2031-50$

[10] Stickler D C 1986 Inverse scattering for stratified elastic media Wave Motion 8 101-12

[11] Carazzone J J 1986 Inversion of P-sv seismic data Geophyiscs 51 1056-68

[12] Wilcox C H 1964 Electric wave propagation on non-uniform coupled transmission lines SIAM Rev. 6 148-65

[13] Bruckstein A M and Kailath T 1987 On discrete Schrödinger equations and their two-component wave equation equivalents J. Math. Phys. 28 2914-24

[14] Case K M and Kac M 1973 A discrete version of the inverse scattering problem J. Math. Phys. 14 594603

[15] Delsarte P and Genin Y 1988 Multichannel singular predictor polynomials IEEE Trans. Circuits Syst. CS-35 190-200

[16] Delsarte P and Genin Y 1986 The split Levinson algorithm IEEE Trans. Acoust. Speech Signal Process. ASSP-34 470-8

[17] Delsarte $\mathrm{P}$ and Genin Y 1987 On the splitting of classical algorithms in linear prediction theory $I E E E$ Trans. Acoust. Speech Signal Process. ASSP-35 645-53

[18] Bistritz Y, Lev-Ari H and Kailath T 1986 Immittance domain Levinson algorithms Proc. 1986 Int. Conf. on Acoustics Speech and Signal Processing (Piscataway, NJ: IEEE) pp 253-6

[19] Newton R G 1980 Inverse scattering. I. One dimension J. Math. Phys. 21 493-505

[20] Bruckstein A M and Kailath T 1987 Inverse scattering for discrete transmission-line models SIAM Rev. 29 359-89 